\title{
Polymer-cholesteric liquid-crystalline composites with a broad light reflection band
}

Michel Mitov

Citation: AIP Conference Proceedings 1736, 020004 (2016); doi: 10.1063/1.4949578

View online: https://doi.org/10.1063/1.4949578

View Table of Contents: http://aip.scitation.org/toc/apc/1736/1

Published by the American Institute of Physics

\section{Articles you may be interested in}

The effect of geometric and electric constraints on the performance of polymer-stabilized cholesteric liquid crystals with a double-handed circularly polarized light reflection band

Journal of Applied Physics 104, 033539 (2008); 10.1063/1.2968245

Switchable broadband light reflection in polymer-stabilized cholesteric liquid crystals Journal of Applied Physics 90, 1730 (2001); 10.1063/1.1388172

Broadband reflective liquid crystalline gels due to the ultraviolet light screening made by the liquid crystal Applied Physics Letters 89, 251907 (2006); 10.1063/1.2416251

Thermally bandwidth-controllable reflective polarizers from (polymer network/liquid crystal/chiral dopant) composites

Applied Physics Letters 82, 2407 (2003); 10.1063/1.1567809

Cholesteric liquid crystal/polymer dispersion for haze-free light shutters

Applied Physics Letters 60, 3102 (1992); 10.1063/1.106765

Fast electro-optic switching in liquid crystal blue phase II

Applied Physics Letters 98, 131905 (2011); 10.1063/1.3564896 


\title{
Polymer-Cholesteric Liquid-Crystalline Composites with a Broad Light Reflection Band
}

\author{
Michel Mitov \\ Centre d'Elaboration de Matériaux et d'Etudes Structurales, CEMES, CNRS, 29 rue J. Marvig, \\ F-31055 Toulouse cx 4, France
}

\begin{abstract}
Cholesteric liquid crystals selectively reflect the light. The reflection bandgap is typically limited to $100 \mathrm{~nm}$ in the visible spectrum and, at the best, $50 \%$ of the unpolarized incident light is reflected. Solutions are found in biopolymers and polymer-liquid crystal composite materials to go beyond these limits.
\end{abstract}

Keywords: cholesteric liquid crystals, chirality, polymer-liquid crystal composites, polymer networks.

PACS: 83.80.Xz, 81.05.Qk, 42.70.-a, 42.70.Df.

\section{INTRODUCTION}

The chiral cholesteric-liquid-crystalline structure, which concerns the organization of chromatin, collagen, chitin, or cellulose, is omnipresent in living matter [1]. In technology, it is found in temperature and pressure sensors, supertwisted nematic liquid crystal displays, optical filters, reflective devices, or cosmetics [2]. The cholesteric liquid crystal (CLC) phase exhibits a helical structure with a twist axis perpendicular to the local director (Fig. 1.a), which comes in whole or in part from the molecular chirality. The cholesteric phase is characterized by two structural parameters: the helical pitch $\mathrm{p}$ and the twist sense. The helical pitch $\mathrm{p}$ gives the distance along the helical axis that corresponds to a rotation of $360^{\circ}$ in the orientation of the rod-like molecules (Fig. 1.b).

When light propagates through a CLC in the Bragg regime, the medium produces interference colors [3]. The light reflection is selective. At normal incidence (for light propagating along the helical axis), the maximum of reflection occurs at the wavelength $\lambda_{0}$ which is directly related to $\mathrm{p}$ by $\lambda_{0}=\mathrm{np}$ where $\mathrm{n}$ is the average refractive index $\left[\mathrm{n}=\left(\mathrm{n}_{\mathrm{o}}+\mathrm{n}_{\mathrm{e}}\right) / 2\right.$, where $\mathrm{n}_{\mathrm{o}}$ and $\mathrm{n}_{\mathrm{e}}$ are the ordinary and extraordinary indices of refraction $]$.
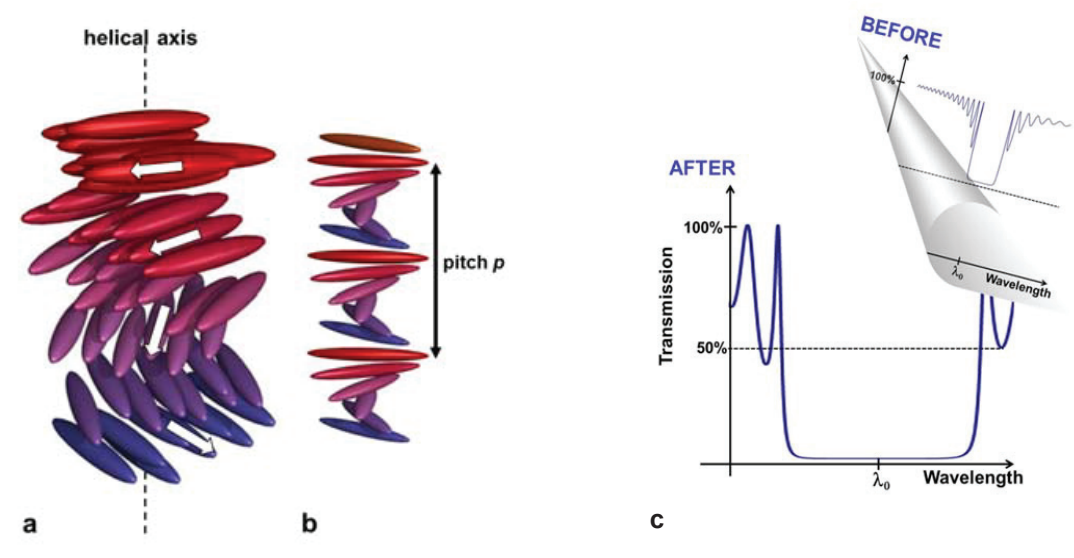

FIGURE 1. (a) Helical structure of rod-like molecules in the cholesteric phase [Adapted with permission from a figure by C. J. Barrett, McGill Univ., Montreal, Canada, and K. G. Yager, Brookhaven National Lab, NY, USA]. (b) A complete revolution occurs over a length equal to the helical pitch. (c) Schematic representation of the present research topic: the selective reflection bandgap is replaced with a broadened bandgap (broad wavelength gap and reflectance above $50 \%$ ).

\footnotetext{
VIII International Conference on "Times of Polymers and Composites" AIP Conf. Proc. 1736, 020004-1-020004-4; doi: 10.1063/1.4949578 Published by AIP Publishing. 978-0-7354-1390-0/\$30.00
} 
At normal incidence, the bandwidth $\Delta \lambda$ is related to the birefringence $\Delta \mathrm{n}\left(\Delta \mathrm{n}=\mathrm{n}_{\mathrm{e}}-\mathrm{n}_{\mathrm{o}}\right)$ and $\mathrm{p}$ by: $\Delta \lambda=\mathrm{p} \Delta \mathrm{n}$. $\Delta \lambda$ is limited to a few tens of nanometers in the visible spectrum because the birefringence is typically limited to 0.5 . The reflectance is equal to at most $50 \%$ for unpolarized incident light, which is a consequence of the polarizationselectivity rule. These limits must be exceeded (Fig. 1.c) for innovative applications like polarizer-free reflective displays, broadband polarizers, optical data storage media, polarization-independent devices, stealth technologies, or smart switchable windows to control solar light and heat. Novel cholesteric-liquid-crystalline architectures with the related fabrication procedures must therefore be developed. This target is the topic of the review paper we have published in 2012 [4].

\section{BROAD WAVELENGTH BANDGAP}

CLCs with a helical pitch gradient are found in living matter [1]. The cuticle of crustaceans and insects is a composite material made of an organic matrix, mainly proteins and chitin (a linear polymer of acetylglucosamine), and a mineral (mainly calcite). After decalcification the chitin fibrils exhibit a twisted organization, which is nothing else than the biological version of the CLC structure. The cholesteric organization is found in the organic matrix of many beetles and is responsible for their iridescent colors. The cuticle of the beetle Plusiotis boucardi exhibits reflections in the green and red [5]. Investigation of cross-sections of the elytra by transmission electron microscopy (TEM) has revealed these reflections come from two CLC structures with distinct pitches. The structure of the cuticle of the crab Carcinus mcenas exhibits a pitch gradient [6]. The distribution of the pitch varies greatly, even in the most regular regions, from 0.25 to $10 \mu \mathrm{m}$, and reflection mainly occurs in the IR. TEM investigations of pitch gradient structures in several insect cuticles have been reported [1].

In man-made materials, most fabrication techniques of graded-pitch structures (Ref. [4] and references therein) use the photopolymerization of monomers mixed with a non-reactive CLC. A UV gradient generates a concentration gradient in chiral species, which is claimed as responsible for a graded-pitch helix and thus bandgap broadening [7]. However the mechanical role of the structure-gradient polymer network over the CLC was ignored until recently. In fact, it is the stress gradient exerted by the network over the CLC structure which basically drives the broadening [8], as evidenced by the absence of a gradient in chiral species. Model calculations and simulations show that the network acts as a spring with a stiffness gradient.
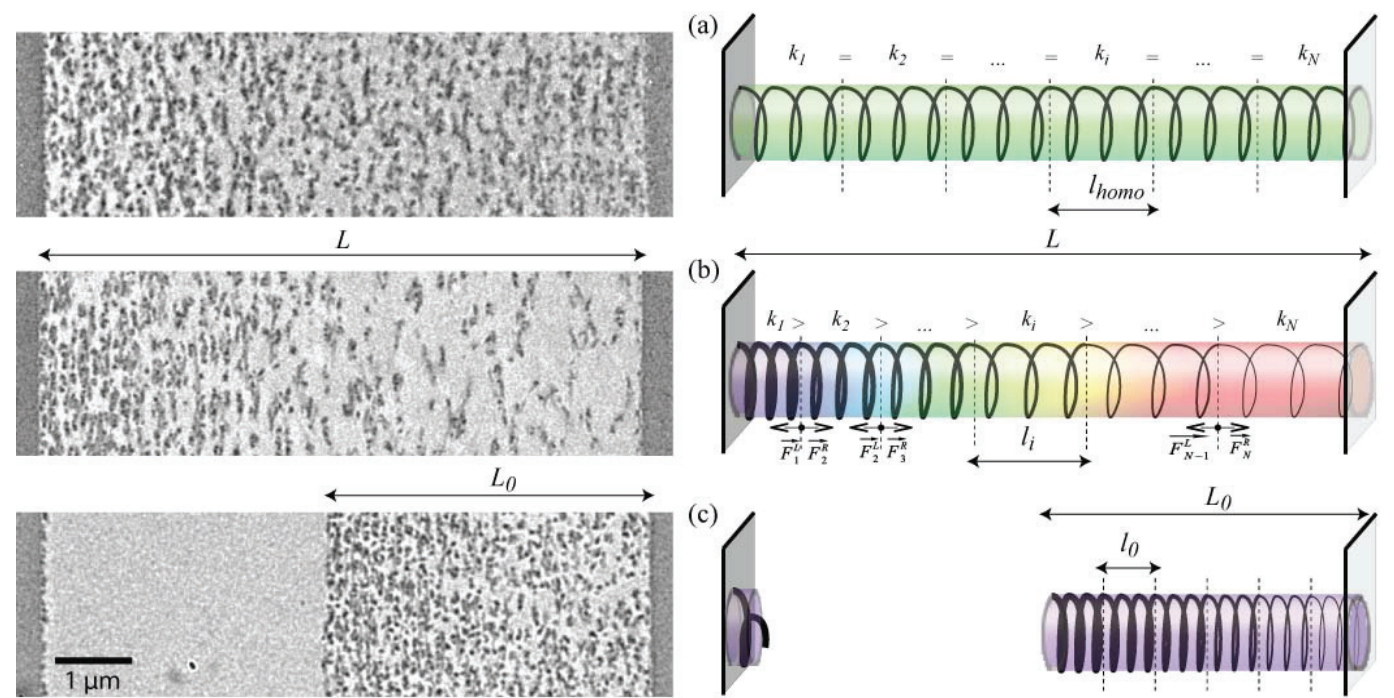

FIGURE 2. Model of coil spring with a gradient of stiffness as inspired by cross-sectional views of the polymer network by TEM. Left column: (a) The network concentration is homogeneously distributed in the bulk (symmetrical irradiation conditions). (b) A gradient of network concentration occurs (asymmetrical conditions). (c) The network has unhooked from one side of the cell and relaxed into the LC volume. This contraction is an experimental evidence of the presence of the restoring force of the spring-network. Right column: Model composed of N sub-springs placed end to end, which represent the helical network. 
(a) The sub-springs have the same spring constant $k_{i}$ and the same length. (b) More concentrated is the network, larger is the constant of the sub-spring locally. In steady state, the springs with the smallest stiffnesses (right part of the gel 'tube') are more elongated than the others (left part). Due to the network which templates the LC structure, the pitch of the gel is proportionally distorted. (c) The sub-springs have relaxed up to their unstressed configuration. They have the same length.

\section{INCREASED REFLECTANCE}

To our knowledge, Plusiotis resplendens is the only beetle whose cuticle reflection properties do not obey the polarization-selectivity rule [9]. The chitin-made cuticle is a three-layer system (Fig. 3) which exhibits, from the top to the bottom of the shell: a left-handed $(\mathrm{LH})$ cholesteric structure, a half-wave-plate nematic structure, and again a LH helix.

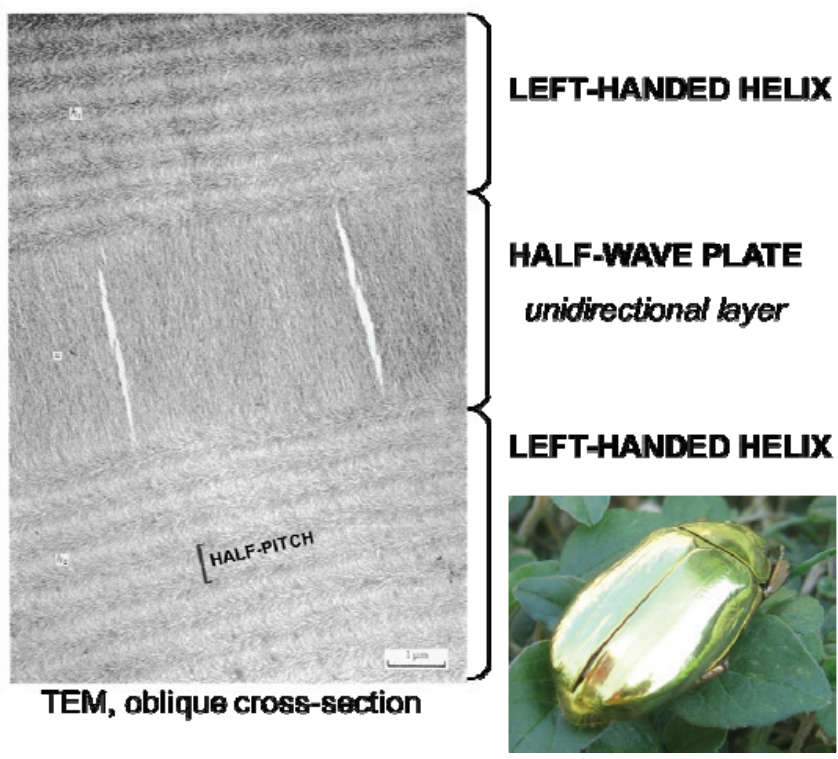

FIGURE 3. TEM micrograph of oblique section of cuticle of Plusiotis resplendens including unidirectional layer and adjacent left-handed layers. The layering periodicity is related to the half pitch. Adapted with permission from [9]. (C) 1971, The Royal Society. Inset: Plusiotis resplendens beetle (by M. Mitov, CNRS-CEMES).

Under unpolarized light conditions, LH circularly-polarized (LHCP) is reflected by the first layer whereas righthanded CP (RHCP) light is transmitted; after passing through the half-wave plate, RHCP light is converted to LHCP light, which is reflected by the third layer; it finally emerges from the cuticle as RHCP light after passing back through the half-wave plate again. Such a dual CP reflector operates between 520 and $640 \mathrm{~nm}$.

Polymer-CLC composite monolayers in which both handed helices coexist in the IR spectrum may be fabricated (Fig. 4) $[10,11]$. Photopolymerizable monomers are introduced in the volume of a CLC exhibiting a thermallyinduced helicity inversion and the blend is then UV-cured when the helix is RH. Due to memory effects brought by the polymer network, the reflectance exceeds 50\% when measured at the temperature assigned at a cholesteric helix with the same pitch but a LH sense before reaction. 

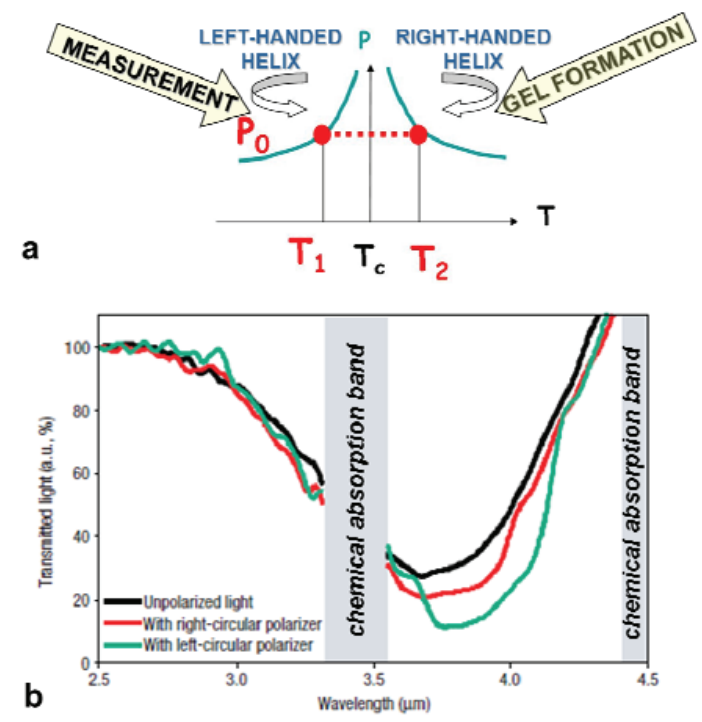

FIGURE 4. (a) Fabrication procedure of a cholesteric gel which exhibits both helicity senses. The gel formation occurs at a temperature $T_{2}$ for which the cholesteric structure of the mixture has a pitch equal to $p_{0}$ and is right handed. The measurement is done at $T_{1}$ for which the free fraction of the gel exhibits a left-handed structure with a pitch close to $p_{0}$. (b) Transmittance of the gel when the incident light is unpolarized or when a left- or right-handed circular polarizer is introduced into the light beam.

Whatever the polarization of the incident light, the reflectance of the cholesteric layer exceeds $50 \%$.

\section{CONCLUSION}

Both reflection limits of CLCs may be exceeded by choosing one or several of the following experimental strategies: stacking layers; driving a thermally induced diffusion in a bilayer to generate a pitch gradient; generating memory effects in polymer-CLC composites; generating a pitch gradient by photopolymerization in itself; using a template. For applications, the solution to choose depends notably on: the desired function; the device in which the layer is implemented; and the fabrication costs for mass production. These composites have potential for use in challenging technological applications, not yet available in everyday life, such as intelligent reflective windows for energy efficiency and for dynamic solar control. CLC structures in living matter with a broad reflection band raise questions, sometimes unsolved, about the morphogenesis of biological tissues, the structural coloration of arthropods for biological functions [dispatch of information (recognition, camouflage), protection against radiation (to avoid overheating)], and its role in evolutionary processes.

\section{REFERENCES}

1. C. Neville, Biology of Fibrous Composites, Cambridge: Cambridge University Press, 1993.

2. Liquid Crystals, edited by S. J. Woltman, G. D Jay and G. P. Crawford, Singapore: World Scientific, 2007.

3. H. Kelker and R. Hatz, Handbook of Liquid Crystals, Weinheim: Verlag Chemie, 1980, pp. 293-337.

4. M. Mitov, Adv. Mater. 24, 6260-6276 (2012).

5. S. A. Jewell, P. Vukusic and N. W. Roberts, New J. of Phys. 9, 1-10 (2007).

6. Y. Bouligand, "Biological analogs of liquid crystals", in Liquid Crystals, edited by L. Liébert, Solid State Physics Suppl. 14, 259-294 (1978).

7. D. J. Broer, J. Lub and G. N. Mol, Nature 378, 467-469 (1995).

8. G. Agez, S. Relaix and M. Mitov, Phys. Rev. E 89, 022513 (2014).

9. S. Caveney, Proc. Roy. Soc. Lond. B 178, 205-225 (1971).

10. M. Mitov and N. Dessaud, Nature Mater. 5, 361-364 (2006).

11. M. Mitov and N. Dessaud, Liq. Cryst. 34, 183-193 (2007). 